\title{
Mother and Baby Units matter: improved outcomes for both
}

\author{
Lucy A. Stephenson, Alastair J. D. Macdonald, Gertrude Seneviratne, Freddie Waites and Susan Pawlby
}

\section{Background}

Mother and Baby Units (MBUS) are usually preferred by patients and clinicians. Current provision is limited, although expansion is in progress. To ensure successful investment in services, outcome measurement is vital.

\section{Aims}

To describe maternal outcomes, mother-infant outcomes and their relationship in one MBU.

\section{Method}

Paired maternal Brief Psychiatric Rating Scale (BPRS) scores, Health of the Nation Outcome Scales (HoNOS) scores and Crittenden CARE-Index (CCI) mother-infant interaction data were collected at admission and discharge.

\section{Results}

There were significant improvements in BPRS $(n=152)$, HoNOS $(n=141)$ and $\mathrm{CCl}(n=62)$ scores across diagnostic groups. Maternal BPRS scores and mother-infant interaction scores were unrelated. Improvement in maternal HoNOS scores was associated with improved maternal sensitivity and reduction in maternal unresponsiveness and infant passiveness.

\section{Conclusions}

Positive outcomes were achieved for mothers and babies across all diagnostic groups. Reduction in maternal symptoms, as measured by BPRS, does not necessarily confer improvement in mother-infant interaction. MBU treatment should focus on both maternal symptoms and mother-infant interaction.

\section{Declaration of interest}

None.

\section{Keywords}

Mother and Baby Unit; outcome studies; perinatal psychiatry; mother-infant interaction.

\section{Copyright and usage}

(C) The Royal College of Psychiatrists 2018. This is an Open Access article, distributed under the terms of the Creative Commons Attribution-NonCommercial-NoDerivatives licence (http://creativecommons.org/licenses/by-nc-nd/4.0/), which permits noncommercial re-use, distribution, and reproduction in any medium, provided the original work is unaltered and is properly cited. The written permission of Cambridge University Press must be obtained for commercial re-use or in order to create a derivative work.
In the UK, failure to address perinatal mental health problems costs approximately $£ 8.1$ billion for each one-year cohort of births - 72\% of which is due to the long-term adverse effects of perinatal mental health problems on child well-being. ${ }^{1,2}$ This suggests that effective services targeting perinatal mental health will have a lasting, trans-generational influence. Psychiatric Mother and Baby Units (MBUs) provide specialist in-patient care for mothers who experience severe mental illness (SMI) without separation from their baby. There are only 17 such units in the UK. This is insufficient to provide for all perinatal women with an SMI who require hospital admission. Thus, women who are eligible for admission are instead separated from their baby and admitted to non-specialist general wards. It is estimated that the number of available MBU beds would need to increase by $50 \%$ to ensure adequate provision. ${ }^{2}$ MBUs are recommended in National Institute for Health and Care Excellence guidelines for women who need admission for psychiatric care in pregnancy or within 12 months of childbirth, and are preferred by patients. ${ }^{3}$ As part of the Five Year Forward View, NHS England have agreed to invest $£ 365$ million to provide four more MBUs in England and to expand the number of specialist community mental health teams. ${ }^{4}$ It has been established that perinatal mental disorders may be associated with poorer outcomes for infants and children. However, the transmission pathway for these difficulties is complex and is not ameliorated by addressing the maternal illness alone. ${ }^{5-7}$ To ensure the investment in perinatal services has the intended long-term results, interventions must be focused on achieving demonstrably positive outcomes for mothers and babies. Parenting skills are a known, accessible and modifiable target in the complex pathway from perinatal SMI to poor outcomes in offspring. ${ }^{7}$ MBUs are ideal facilities to offer holistic interventions that address maternal mental illness and parenting issues. However, owing to the variety of MBUs and their interdisciplinary nature, patient outcomes are complex to evaluate. So far, MBUs have largely been evaluated according to patient preference and process (e.g. whether mother and child stay together) rather than outcome (e.g. a measured improvement in maternal psychopathology or mother-infant interaction). This study explores the extent to which admission to an MBU involving specific motherinfant relationship support is associated with change in routine maternal mental health measures as well as in mother-infant interaction.

The study aims to test three hypotheses.

(a) Following MBU admission, there will be a demonstrable improvement in maternal mental health using routine clinical outcome measures.

(b) Mothers discharged from the $\mathrm{MBU}$ will be significantly more sensitive and less unresponsive to their infants than at admission.

(c) Their infants will be significantly more cooperative and less passive than at admission.

\section{Method}

This study took place in a 13-bedded MBU in South London. It is the largest in the UK and serves an ethnically and socioeconomically diverse population from some of the most deprived parts of London. ${ }^{8}$ Pregnant women and mothers of babies under 12 months old who experience SMI receive intensive treatment and support from a specialised multidisciplinary team; the majority (88\%) are admitted postnatally with their baby. Some mothers without active SMI but who may have a diagnosis of borderline 
personality disorder are also admitted for brief parenting assessments. Some mothers who are in remission, but have an established or previous perinatal history of SMI, are admitted for prophylactic observation and support. Each mother and her baby have their own room, and a nursery is available for overnight care when required.

The unit provides an individualised treatment programme from the multidisciplinary team, including nursery nurses. Care plans involve antenatal and postnatal care for the mother and the baby, management of maternal SMI, monitoring of physical health, appropriate pharmacological interventions suitable for pregnant women and breastfeeding mothers, developmental assessment of babies, intensive mental health nursing, occupational therapy and support for developing independent living skills, and assessment of and assistance with social needs. Psychological therapies are available for mothers, and for mothers with their infants. The latter includes video feedback sessions (mean of two sessions) to enhance mothers' recognition of their infants' cues and sensitive caregiving. ${ }^{9}$ The MBU environment is a highly specialist acute psychiatric ward, where mothers who are extremely unwell come with their infants for a relatively short period of time. More intensive mother-infant relationship psychotherapy and other similar interventions are offered to the mothers on a longer-term out-patient basis when they are no longer experiencing acute mental illness on the psychotic spectrum.

\section{Procedure and measures}

All admissions from April 2012 to December 2014 were eligible for inclusion in the study. Mothers were excluded if they were only admitted for a parenting assessment; mothers admitted before delivery who returned directly from the labour ward were included. When mothers were readmitted during the study period, the longest spell was included in the analysis. As part of routine outcome measurement on the MBU, the Brief Psychiatric Rating Scale (BPRS) ${ }^{10}$ was completed on admission and discharge by two of the authors (G.S. and L.S.) and other psychiatrists trained in its use. This is an 18-item scale of psychopathology in SMI with a maximum score of 128, divided into five subscales - affect, positive symptoms, negative symptoms, resistance and activation. ${ }^{11}$ Some BPRS ratings were completed retrospectively by consensus on case notes review by two authors (L.S. and G.S.). In order that the improvement in BPRS scores was more clinically meaningful, we followed Leucht et al's ${ }^{12}$ categorisation of scores on admission and discharge: $0-30=$ none or mild, $31-50=$ moderate, and $\geq 51=$ severe disorder; an improvement over the admission of at least $30 \%$ was considered by Leucht to be 'minimal' and $50 \%$ or more 'marked'.

At the same time, the Health of the Nation Outcome Scales $(\mathrm{HoNOS})^{13}$ were completed as part of routine outcomes measurement and captured using the Clinical Record Interactive Search (CRIS) system, which is derived from electronic patient records. ${ }^{14}$ These are twelve clinician-rated scales, rated by all qualified staff, covering psychiatric symptoms, functioning and social circumstances. Each scale is rated 0 for no problem; 1 for a problem that would not normally need intervention; and 2, 3 and 4 corresponding to a mild, moderate or severe problem, respectively, in that domain. Relationship problems included the rating of the quality of the relationship with the baby as well as with others. Three main problems were categorised under 'Other mental and behavioural problems': mental strain and tension, anxiety and sleep. At the time of the study, there were regular training sessions in using HoNOS for all staff. By consensus, local clinical practice has established that moving across the $2 / 3$ cut-off point in any HoNOS scale represents clinically significant change; these moves were examined, as well as any change in mean total HoNOS scores.
All mothers admitted to the MBU are routinely offered video feedback sessions in the first week of admission and after their final discharge meeting. This is an intervention targeted at assessing and improving mother-infant interaction. ${ }^{9}$ A developmental psychologist (S.P.) and two nursery nurses, all of whom had been trained in using the Crittenden CARE-Index (CCI), rated the video recordings.

The CCI comprises several subscales that measure maternal sensitivity, control, unresponsiveness and infant cooperativeness, compulsiveness, difficultness and passiveness. All raters were trained to reach $80 \%$ agreement on each scale. Differences in coding were discussed and consensus scoring reached. A fourth rater coded nine $(15 \%)$ of the play sessions of the 62 participants. The interrater intraclass correlation coefficients (ICC) for both maternal sensitivity and infant cooperativeness were 0.78 , indicating acceptable interrater reliability. Although ratings were undertaken by staff who in some cases knew the patients clinically, the independence of ratings was somewhat assured by a time lapse of several months before the ratings were carried out. The fourth rater was unaware of any of the maternal diagnoses. Behaviour in both mother and infant was assessed, although the focus was on the interaction rather than either individual. The domains of interest included maternal sensitivity and unresponsive behaviour, as well as infant cooperative and passive behaviour. These measures are independent of infant development and have been deemed appropriate in assessing the effectiveness of an intervention. ${ }^{9,15}$ Participants were excluded from this analysis if only one video was available, if the mother was not currently unwell, or if the mother had been admitted to the unit in early pregnancy and had therefore received the majority of her care while on the unit, prior to the baseline, postnatal video of the mother-infant interaction being made.

All mothers were assigned to an ICD-10 diagnosis during their admission by a consultant psychiatrist (G.S.) and were then grouped according to their predominant clinical symptoms. When diagnosis had changed, the final diagnosis was used. In analyses of diagnosis, mothers admitted for prevention who were not clinically unwell were excluded.

\section{Ethics}

The study of mother-baby interactions was approved by the Institute of Psychiatry, King's College London Ethics of Research Committee (reference 05/Q0706/159). Informed written consent was obtained for the recordings of the interactions to be used for research, and all procedures were conducted in accordance with the British Psychological Society ethical guidelines. CRIS was approved as a data-set for secondary analysis by the Oxfordshire Research Ethics Committee C (reference 08/H0606/71). The CRIS oversight committee (reference 17-035) approved the use of routine outcome measurements in this project.

\section{Statistical analysis}

For readmissions, only the longest MBU admission was used in the data analysis.

Distribution and skewness of the BPRS totals, HoNOS totals and CCI were assessed for normality and linearity; if data did not reach assumptions of normality $(<-2$ or $>2)$, non-parametric analyses were used. Non-parametric repeated measures models (Wilcoxon signed-rank test) were used to compare the mothers' BPRS scores and the interaction scores of mothers and babies on admission and on discharge from the MBU, with time as the within-subjects factor. Effect sizes were calculated by dividing the $z$-values by $\sqrt{ } N$, where $N$ is the number of observations (the number of cases $\times 2$ ). Using Cohen's criteria, ${ }^{16} 0.1=$ a small effect, 
$0.3=$ a medium effect and $0.5=$ a large effect. Paired samples $t$-tests were used to assess the differences between the HoNOS scores on admission and discharge, and a univariate general linear model for repeated measures was used to assess the differences between diagnostic groups, with time as the within-subjects' factor and diagnosis as the between-subjects' factor. An independent sample Kruskal-Wallis test was used to compare CCI scores between the diagnostic groups. Independent sample median tests with pairwise comparisons measured differences in BPRS scores between the diagnostic groups. Spearman's rho was reported for correlations between the outcome measures.

\section{Results}

During the study period, 172 mothers were admitted to the MBU. Thirteen women admitted only for parenting assessments rather than treatment for acute mental illness were excluded, leaving a sample of 159 mothers. Of these, 150 were admitted during the baby's first year and nine were admitted before delivery, returning to the MBU from the labour ward. Eight of the mothers were discharged and then readmitted during the study (five after temporary discharge to a psychiatric intensive care unit, two following deterioration after discharge home, and one after temporary transfer to another unit). Admission and demographic data are shown in Table 1. The median admission time was 60 days, ranging from one to 209 days.

Of the 159 mothers for whom the BPRS had been completed at admission and discharge, seven had been admitted prophylactically and had no current psychiatric symptoms. These seven were excluded from the analyses. Seventy-nine mothers consented to CCI observation at admission, and 63 at admission and discharge. One of the 63 women, who was pregnant on admission and gave birth during her admission, was excluded from CCI analysis. CCI analyses were undertaken for the 62 mothers who were admitted with their babies (55\% male; age range on admission 2-325 days) and provided video data at admission and discharge (Table 2).

There were no significant differences in maternal age, ethnicity or diagnostic group between mother-infant dyads who did and did not participate in the video feedback intervention.

\begin{tabular}{|c|c|c|c|c|}
\hline & & Mean & s.d. & Median \\
\hline Age at admission, years & $16-43$ & 30.9 & 5.9 & 31 \\
\hline Length of admission, days & $1-209$ & 67.7 & 42.7 & 60 \\
\hline \multicolumn{5}{|l|}{ Relationship status, $\boldsymbol{N}(\%)$} \\
\hline Married/civil partner & $73(45.9)$ & & & \\
\hline Single & $62(39.0)$ & & & \\
\hline Cohabiting & $15(9.4)$ & & & \\
\hline Separated & $4(2.5)$ & & & \\
\hline Not known & $3(1.9)$ & & & \\
\hline Not disclosed & $2(1.3)$ & & & \\
\hline \multicolumn{5}{|l|}{ Ethnic group, $N(\%)$} \\
\hline White & $79(49.7)$ & & & \\
\hline Black & $44(27.7)$ & & & \\
\hline Asian & 18 (11.3) & & & \\
\hline Mixed & 4 (2.5) & & & \\
\hline Other & $9(5.7)$ & & & \\
\hline Missing & $5(3.1)$ & & & \\
\hline $\begin{array}{l}\text { Diagnostic group at admission, } \\
\qquad N(\%)\end{array}$ & & \multicolumn{3}{|c|}{$\begin{array}{l}\text { Length of admission, } \\
\text { days }\end{array}$} \\
\hline Depression & $80(50.3)$ & 74.6 & 46.6 & 69.5 \\
\hline Mania & $35(22.0)$ & 58.4 & 35.6 & 56 \\
\hline Anxiety/obsessive-compulsive & $20(12.6)$ & 58.7 & 38 & 55.5 \\
\hline Schizophrenia & $17(10.7)$ & 96.5 & 58.2 & 77 \\
\hline No current psychiatric symptoms & $7(4.4)$ & 41.0 & 12.0 & 40 \\
\hline
\end{tabular}

\begin{tabular}{|c|c|c|c|c|}
\hline & & Mean & s.d. & Median \\
\hline Age at admission, days & $2-325$ & 99.0 & 92.3 & 67.5 \\
\hline $\begin{array}{l}\text { Age at discharge, days } \\
\text { Infant gender, } N(\%)\end{array}$ & $48-472$ & 188.6 & 108.3 & 160.5 \\
\hline Male & $34(54.8)$ & & & \\
\hline Female & $28(45.2)$ & & & \\
\hline
\end{tabular}

\section{Maternal mental health as measured by BPRS}

On admission, there was a significant difference in the total BPRS scores of the different diagnostic groups (independent sample median test with pairwise comparisons, $P<0.05$ ), with the mothers with anxiety having significantly lower scores than mothers with mania (pairwise comparisons test statistic $=7.30$, adjusted significance $P=0.04$ ). On discharge, there were no significant differences between the diagnostic groups (independent sample median test with pairwise comparisons, $P=0.26$ ).

\section{Change in BPRS scores from MBU admission to discharge}

Changes in the BPRS total and subscale scores are shown in Table 3.

Overall, there was significant improvement between admission and discharge, at the $P<0.001$ level, in the total BPRS score and in the individual subscales (Wilcoxon signed-ranks test: BPRS total, $z=10.54$; affect, $z=10.23$; negative symptoms, $z=9.29$; positive symptoms, $z=8.85$; activation, $z=10.34$; resistance, $z=8.81$ ). Irrespective of their diagnosis, the mothers' BPRS total and subscale scores improved significantly between admission and discharge. In each case, the effect size was large $(r>0.50)$ or medium to large $(0.30<r<0.50)$.

There was a significant difference in the level of change in the BPRS total score between the diagnostic groups $\left(\chi_{(6)}^{2}=24.59, P<\right.$ 0.001). A higher proportion of mothers with schizophrenia, mania and depression had markedly or minimally improved scores on discharge compared with admission, whereas a higher proportion of mothers with anxiety showed minimal or no improvement from admission to discharge (as shown in Table 4).

\section{Maternal mental health and well-being as measured by the HONOS}

Of the 152 eligible participants 141 mothers (92.8\%) had HoNOS ratings at admission and $113(74.3 \%)$ had ratings at admission and at discharge. There were no significant differences in maternal age, ethnicity or diagnosis between those who had paired HoNOS scores and those who did not.

Mothers' HoNOS scores on admission $(N=141)$ demonstrated a wide range of severe difficulties (rated 3 or 4 on the scale) across behavioural, physical, psychological and social domains. On discharge, the proportion of mothers $(N=113)$ with severe difficulties in each domain as rated on the HoNOS had decreased. On admission, $26 \%$ of mothers $(N=29)$ v. $0.9 \%(N=1)$ on discharge had severe behavioural problems; $9.9 \%(N=14) v .1 .8 \%(N=2)$, respectively, had problems with non-accidental self-injury; none had problems with drinking or substance misuse, as these women would not have been admitted to the MBU; $8.5 \%(N=12)$ v. $0.9 \%(N=1)$ had severe cognitive problems; $9.2 \%(N=13)$ v. $2.7 \%(N=3)$ had a physical or severe disability problem; $34 \%(N=48) v .3 .5 \%(N=4)$ had severe problems with hallucinations or delusions; $45.4 \%(N=64)$ v. $8.8 \%(N=10)$ had severe problems with depressed mood; $68.1 \%(N=96) v .8 .8 \%(N=10)$ had other mental and behavioural problems, mostly associated with anxiety; $22 \%(N=31)$ v. $6.2 \%$ $(N=7)$ had severe problems with relationships; $14.9 \%(N=21) v$. 


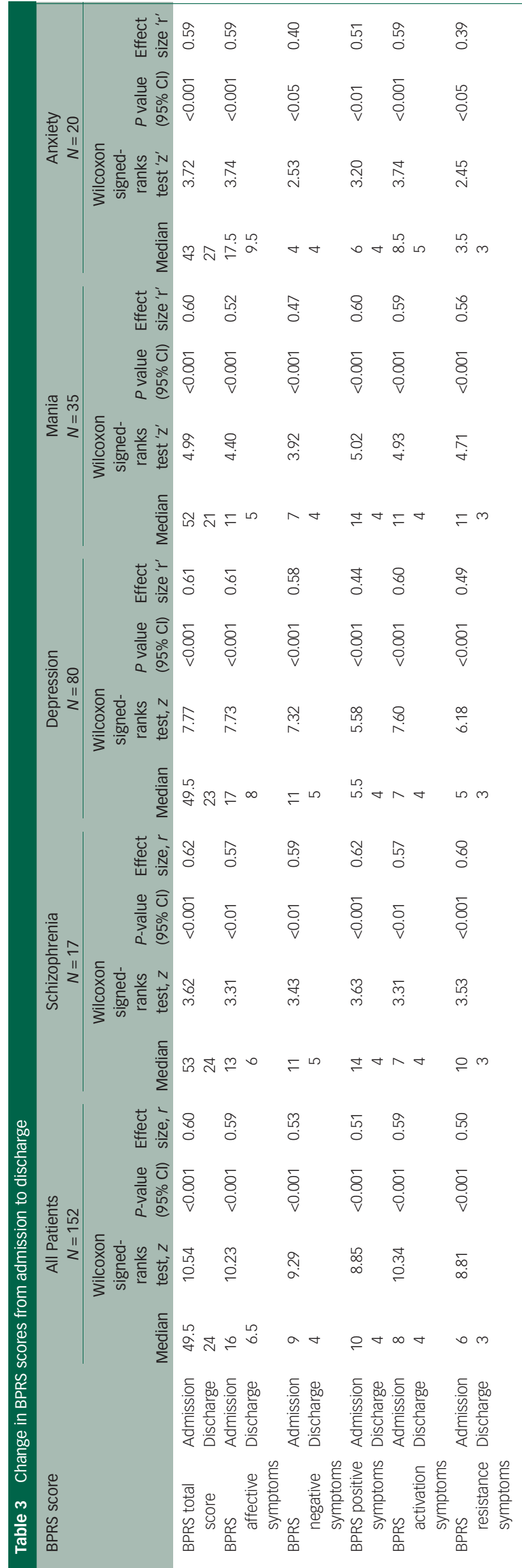

$1.8 \%(N=2)$ had severe problems with activities of daily living; $10.6 \%(N=15)$ v. $8.8 \%(N=10)$ had severe problems with their living conditions; and $11.3 \%(N=16)$ v. $2.7 \%(N=3)$ had severe problems with work or activities.

\section{Changes in HoNOS scores from admission to discharge}

There was a significant improvement $\left(t_{(112)}=17.51 P<0.001\right)$ in mean total HoNOS scores from admission to discharge, from 14.07 (s.d. 5.30) to 5.00 (s.d. 4.83). The HoNOS scores in all domains except that of living conditions improved significantly from admission to discharge: behavioural problems, $\left(\mathrm{t}_{(112)}=8.47\right.$, $P<0.001)$; non-accidental self-injury $\left(t_{(112)}=5.16, P<0.001\right)$; cognitive problems $\left(t_{(112)}=6.80, P<0.001\right)$; physical and disability problems $\left(t_{(112)}=4.24, P<0.001\right)$; hallucinations and delusions $\left(t_{(112)}=9.07, P<0.001\right)$; depressed $\operatorname{mood}\left(t_{(112)}=13.58, P<0.001\right)$; other mental and behavioural problems $\left(t_{(112)}=12.27, P<0.001\right)$; relationship problems $\left(t_{(112)}=5.32, P<0.001\right)$; activities of daily living $\left(t_{(112)}=6.34, P<0.001\right)$; problems with work or activities $\left(t_{(112)}=3.74, P<0.001\right)$; and living conditions $\left(t_{(112)}=0.78, P=\right.$ $0.44)$. There were no significant differences in the level of change in HoNOS scores between the different diagnostic groups.

Figure 1 shows the change in each scale across the $2 / 3$ score cutoff point in mothers rated at admission and discharge.

\section{Change in mother-infant relationship}

The babies' ages on admission ranged from 2 days to 325 days with a median of 67 days. There were no significant correlations between the baby's age on admission and maternal-infant CCI scores on either admission or discharge. The scales of maternal controlling and unresponsiveness were highly and negatively correlated, as were the infant scales of difficultness, compulsiveness and passiveness. Only maternal sensitivity and unresponsiveness and infant cooperativeness and passiveness are presented here.

Table 5 shows changes in both maternal and infant behaviour between admission and discharge. These were explored in a series of repeated measures models. Mothers were significantly more sensitive and less unresponsive, and infants showed more cooperativeness, at discharge than on admission. There was no significant change in the infants' passiveness scores. The changes in maternal sensitivity and infant cooperativeness were positively correlated $\left(r_{(s)}=0.69, P<0.001\right)$

There were no significant differences between the four diagnostic groups (schizophrenia, depression, mania and anxiety) in their ranked scores in maternal sensitivity on admission $\left(\chi_{(3)}^{2}=3.94\right.$, $P=0.27)$ or discharge $\left(\chi_{(3)}^{2}=2.78, P=0.43\right)$, or in the change in scores over time $\left(\chi_{(3)}^{2}=1.01, P=0.80\right)$. Nor were there any differences between the groups in maternal unresponsiveness on admission $\left(\chi_{(3)}^{2}=6.26, P=0.10\right)$ or discharge $\left(\chi_{(3)}^{2}=0.32, P=0.96\right)$, or in the change in scores over time $\left(\chi_{(3)}^{2}=4.12, P=0.25\right)$. Similarly, there were no significant differences between the four diagnostic groups in the ranked scores of the babies' cooperativeness on admission $\left(\chi_{(3)}^{2}=2.71, P=0.44\right)$ or discharge $\left(\chi_{(3)}^{2}=3.97, P=0.26\right)$, in the change in scores over time $\left(\chi_{(3)}^{2}=5.49, P=0.14\right)$, in the babies' passiveness on admission $\left(\chi_{(3)}^{2}=1.35, P=0.72\right)$ or discharge $\left(\chi_{(3)}^{2}=\right.$ 2.66, $P=0.45)$, or in the change in scores over time $\left(\chi_{(3)}^{2}=2.41\right.$, $P=0.49)$. Moreover, there were no significant correlations between the duration of the admission and the changes in the mothers' or babies' interaction scores.

Although the vast majority $(85 \%)$ of mother-infant pairs improved in their dyadic interactions, nine mother-infant pairs had lower scores in maternal sensitivity and infant cooperativeness on discharge than on admission. Compared with those who had higher scores on discharge than on admission $(N=53)$, there were no significant differences in infant gender, maternal diagnosis, 
Table 4 Change in BPRS scores from admission to discharge

\begin{tabular}{|c|c|c|c|c|c|c|c|c|c|c|}
\hline \multirow[t]{2}{*}{ Categorical change } & \multicolumn{2}{|c|}{$\begin{array}{l}\text { All patients } \\
\qquad N=152\end{array}$} & \multicolumn{2}{|c|}{$\begin{array}{c}\text { Schizophrenia } \\
\qquad N=17\end{array}$} & \multicolumn{2}{|c|}{$\begin{array}{l}\text { Depression } \\
\qquad N=80\end{array}$} & \multicolumn{2}{|c|}{$\begin{array}{l}\text { Mania } \\
N=35\end{array}$} & \multicolumn{2}{|c|}{$\begin{array}{l}\text { Anxiety } \\
N=20\end{array}$} \\
\hline & $N$ & $\%$ & $N$ & $\%$ & $N$ & $\%$ & $N$ & $\%$ & $N$ & $\%$ \\
\hline Marked improvement & 65 & 42.8 & 10 & 58.8 & 31 & 38.8 & 22 & 62.9 & 2 & 10 \\
\hline Minimal improvement & 65 & 42.8 & 4 & 23.5 & 42 & 52.5 & 8 & 22.9 & 11 & 55 \\
\hline No improvement or decline & 22 & 14.5 & 3 & 17.6 & 7 & 8.8 & 5 & 14.3 & 7 & 35 \\
\hline
\end{tabular}

maternal age, infant age on admission, infant age on discharge or duration of stay on the MBU. However, compared with the scores of the pairs whose scores improved, the maternal sensitivity and infant cooperativeness scores of the nine pairs whose scores were lower on discharge were significantly higher on admission (maternal sensitivity: $M=4.72$ (s.d. $=2.10) v . M=6.89$ (s.d. $=2.62), t_{(60)}$ $=-2.77, P<0.01$; infant cooperativeness: $M=3.98$ (s.d. $=2.68) v$. $M=7.33$ (s.d. $\left.=2.55), t_{(60)}=-3.49, P=0.001\right)$. Moreover, compared with the maternal sensitivity and infant cooperativeness scores of those whose scores were higher on discharge than on admission

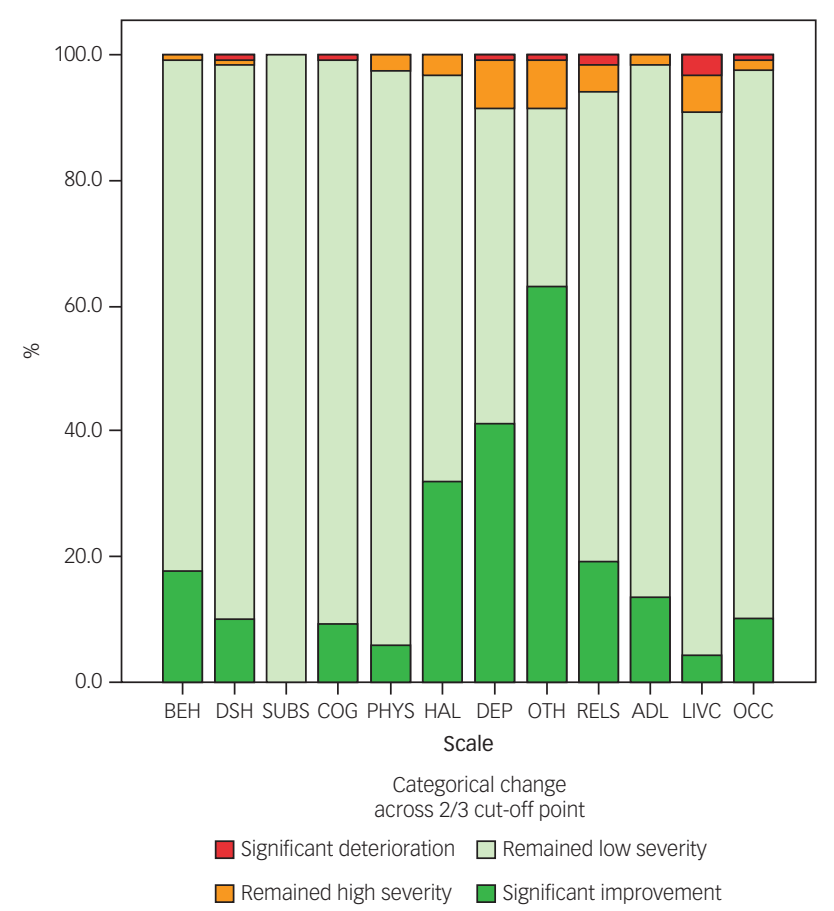

Fig. 1 Categorical change in HoNOS scales' scores from admission to discharge. ADL, problems with activities of daily living; BEH, overactive, aggressive, disruptive or agitated behaviour; COG, cognitive problems; DEP, problems with depressed mood; DSH, non-accidental self-injury; HAL, problems associated with hallucinations or delusions; LIVC, problems with living conditions; OCC, problems with occupation and activities; OTH, other mental and behavioural problems (e.g. anxiety); PHYS, physical illness or disability problems; RELS, problems with relationships; SUBS, problem drinking or drug taking.
$(N=53)$, those whose scores had decreased $(N=9)$ had significantly lower discharge scores (maternal sensitivity: $M=7.40$ (s.d. $=2.57$ ) v. $M=4.11$ (s.d. $=2.57), t_{(60)}=3.55, P=0.001$; infant cooperativeness: $M=6.40$ (s.d. =2.61) v. $M=4.33$ (s.d. =2.83), $\left.t_{(60)}=2.17, P=0.03\right)$.

\section{Relationship between maternal psychiatric ratings and mother-infant interaction}

There were expected positive correlations between HoNOS and BPRS scores at admission $\left(N=141, r_{(\mathrm{s})} 0.19, P<0.05\right)$ and on discharge $\left(N=113, r_{(s)}=0.54, P<0.001\right) .{ }^{17}$ There were no significant correlations in the amount of change between the HoNOS and BPRS scores.

Sixty-two mothers had BPRS and CCI scores. There were no significant correlations between the BPRS scores and the CCI scores on admission or discharge, or in the changes during the admission period.

Sixty mothers had HoNOS and CCI scores at admission: a significant positive correlation between the total HoNOS score and infant passiveness $\left(N=60, r_{(s)}=0.26, P<0.05\right)$ was found, but there were no other significant correlations. There were no significant correlations between the total HoNOS scores and the CCI on discharge. However, there were significant correlations between the improvement in the HoNOS scores over the admission and the improvement in the CCI scores for maternal sensitivity $(N=49$, $\left.r_{(\mathrm{s})}=-0.36, P=0.01\right)$; maternal unresponsiveness $\left(r_{(\mathrm{s})}=+0.44\right.$, $P=0.001)$; and infant passiveness $\left(r_{(\mathrm{s})}=+0.28, P<0.05\right)$. There was no significant correlation between the change in HoNOS score and infant cooperativeness. However, there were significant correlations between improvement in some of the HoNOS domains and the CCI scores. Less overactive, aggressive behaviour was correlated with less maternal unresponsive interaction with the infant $\left(r_{(\mathrm{s})}=+0.28, P<0.05\right)$. Fewer problems with depressed mood were correlated with less maternal unresponsive interaction $\left(r_{(s)}=+0.45, P=0.001\right)$ and less infant passiveness $\left(r_{(s)}=+0.48\right.$, $P<0.001)$. A reduction in other mental and behavioural symptoms, such as anxiety and sleep problems, was correlated with an increase in maternal sensitivity $\left(r_{(\mathrm{s})}=-0.30, P<0.05\right)$ and a reduction in maternal unresponsiveness $\left(r_{(\mathrm{s})}=+0.31, P=<0.05\right)$.

\section{Discussion}

The findings of this observational study broadly support our hypotheses. Following MBU admission, there was a significant improvement in mental health and mother-infant interaction,

Table $5 \mathrm{CCl}$ scores at admission and discharge

\begin{tabular}{|c|c|c|c|c|c|}
\hline & $N$ & Admission, mean (s.d.) & Discharge, mean (s.d.) & $Z$ & $P$-value \\
\hline Maternal sensitivity & 62 & $5.03(2.29)$ & $6.92(2.80)$ & -4.27 & $<0.001$ \\
\hline Maternal unresponsiveness & 62 & $3.11(2.94)$ & $2.13(2.47)$ & -2.34 & 0.02 \\
\hline Infant cooperativeness & 62 & $4.47(2.90)$ & $6.10(2.72)$ & -3.77 & $<0.001$ \\
\hline Infant passiveness & 62 & $3.40(3.20)$ & $3.06(2.67)$ & -0.48 & 0.63 \\
\hline
\end{tabular}


with few significant differences between diagnostic groups. Both BPRS and HoNOS scores demonstrated significant improvement in mental health symptoms across a range of severe psychiatric disorders. These disorders were divided into four diagnostic groupings, depending on the mother's most prominent symptom profile schizophrenia, depression, mania and anxiety. Mothers with anxiety had fewer symptoms on the BPRS on admission compared with mothers with mania, but by discharge there were no differences in their BPRS scores. All diagnostic groups had significantly fewer psychiatric symptoms on discharge compared with admission, with a large or medium-to-large effect size on all the BPRS subscales: affect, negative symptoms, positive symptoms, activation and resistance. Similarly, mothers' scores on the HoNOS improved during their admission to the MBU, with very few women having severe difficulties in any domain on discharge. Although the BPRS and HoNOS scores were significantly correlated, we found no association between a reduction in psychiatric symptoms as rated by the BPRS and the dyadic interaction between the mother and her infant. However, overall there were significant associations between the reduction in HoNOS scores and improvement in mother-infant interaction. In particular, less overactive, aggressive behaviour was associated with less unresponsive behaviour towards the infant, as were fewer problems with depressed mood. The latter was also associated with less infant passive behaviour. A reduction in behavioural symptoms such as anxiety and sleep was associated with increased maternal sensitivity and reduced maternal unresponsiveness towards the infant.

Outcomes from this MBU suggest that treatment of both a mother's illness and support of her relationship with her baby are helpful for women with a wide variety of mental illnesses. They support the importance of nursery nurses on an MBU, whose job it is to help increase mothers' confidence and competence in their skills as parents. We have shown that however severe the mother's illness, and whatever her diagnosis, she is highly likely to experience a reduction in symptoms over the course of her admission and an improvement in her interaction with her baby, becoming more sensitive and less unresponsive. Moreover, the baby is also likely to show improved interaction with his or her mother in terms of cooperativeness, thus improving the dyadic synchrony of the interaction. Further observations of the mother-infant interaction following discharge would be needed to establish whether the observed lack of the expected reduction in the passive dimension of the CCI is a stable finding. However, there was a small group of mother-infant pairs (15\%) where we observed a decrease in the sensitivity and cooperativeness scores of the dyad during their MBU admission. Although we could not find any demographic factors, such as length of admission, that predicted this decrease, we did find that these dyads had higher scores on admission than the group whose scores improved and lower scores on discharge. More video recordings of play sessions between the dyads would give us the opportunity to understand these differences and to ascertain the stability of the dyadic interaction. Furthermore, information on secondary diagnoses and comorbidity, with particular reference to personality disorders, might elucidate this finding. Two of the nine dyads (22.2\%) whose CCI scores were lower at discharge compared with admission had a secondary diagnosis of emotionally unstable personality disorder (EUPD), whereas this was the case for only two out of the 53 dyads (3.8\%) whose scores were higher. A larger number of participants would be needed to confirm whether dyads where the mothers had a diagnosis of EUPD had less stable interaction patterns.

We believe this to be the first account of routine clinical outcome measurements in mothers together with study of mother-infant interactions in an MBU, an approach suggested by Christl et al (2015). ${ }^{18}$ Previously, Kumar et al $(1995)^{19}$ reported outcomes of 100 consecutive admissions to the same unit in terms of length of admission, reliance on medication and whether separation from the infant was necessary at discharge. These process measurement approaches to evaluation have been reported elsewhere, ${ }^{20-23}$ but they suggest that outcome quality is at least as important as process quality measurement. ${ }^{24}$

The finding that improvement in the mother's mood is associated with an improvement in the infant's directly observed cooperative behaviour supports our previous findings. ${ }^{9}$ It is interesting that although the BPRS and HoNOS scores at admission were correlated, improvement in the dyadic interaction between the mother and her infant was only correlated with a reduction in HoNOS and not BPRS scores. This may reflect the difference between the focus of the HoNOS scores on a wealth of multidisciplinary information on the mother's overall well-being, including behaviour, psychiatric symptoms, cognitive abilities, relationships, activities of daily living, work, activities and living conditions, compared with the focus of the BPRS on purely psychiatric symptoms. The evidence reported here confirms that admission to an MBU where treatment and direct support for both mother and infant, separately and together, are provided results in improved maternal psychiatric and general well-being, as well as an improved relationship between mother and baby.

The average length of stay in this UK MBU study was 68 days with a median of 60 days. Although long, and therefore expensive, this was no longer than that described by $\mathrm{Kumar}^{19}$ or Abel et al (2005). ${ }^{25}$ The length of admission was also not very different from the 2016-2017 lengths of stay for schizophrenia (100 days) or mood disorders (40 days). ${ }^{26}$ It is, however, in contrast to the shorter admission times of 15-27 days described in MBUs in Australia. $^{21}$

Currently, discharge from an in-patient MBU depends largely on the reduction in the mother's psychiatric symptoms and her ability to cope at home and in the outside world. If a mother is seen to be unable to care for her baby, then she may be discharged from the MBU with a family member or another person taking on the role of primary caregiver. Our study supports the view that the mother's relationship with and care of her baby should be a priority in the treatment offered to MBU patients, along with the reduction of psychiatric symptoms, so that separation on discharge does not occur (as was the case for all the dyads described here) and mothers go home as primary carers for their babies with whom they have developed strong, positive relationships.

This study has the limitations of any observational one, and so no causal relationships are immediately justified. In the 'holy triad' of understanding of outcomes, only context and outcomes data were available; interventions were not trapped. The study is limited to one MBU and shows no significant differences between diagnostic groupings. This latter finding could be attributed to the small sample size and to the differing number of women in the different groups. Only larger studies that include other MBUs could provide an answer to the question of whether there are differences in outcome for mothers with different diagnostic profiles. Furthermore, the analyses of the dyadic interaction between the mother and infant rely on only one play session on admission and on discharge. Observations of more than one play session on admission and discharge would allow us to look at the stability of the dyad's interactions. Moreover, we do not know whether the improvements in mother-infant interactions observed over the admission time are maintained post discharge. There is some preliminary evidence from the same MBU that shows a secure attachment in the second year of life among dyads who received a mindmindedness video feedback intervention. ${ }^{27}$

This study does, however, have many strengths: the systematic recording of psychiatric measures, the BPRS and HoNOS, by 
different trained members of the multidisciplinary team over a 2-year period; the systematic observation of mother-infant interaction and the use of video feedback with the mothers in order to support their relationships with their babies; and the coding of the video of the mother-infant interaction sessions using the CCI, by trained observers blind to the mother's diagnosis.

Further research based on the 'proof of concept' of the mindmindedness intervention ${ }^{27}$ is in the pipeline, along with ESMI (Effectiveness and cost-effectivenesS of Mother and baby units $v$. general psychiatric In-patient wards and crisis resolution team services; https://www.kcl.ac.uk/ioppn/depts/hspr/research/CEPH/ wmh/projects/A-Z/ESMI-MBU.aspx). Future research should also consider contrasting outcomes for dyads who have been admitted to different MBUs, some of which use video-feedback intervention and some that do not.

Perinatal mental illness is a global public health priority. This paper adds weight to calls for universally accessible MBU provision, where mothers with SMI can be treated alongside their babies and good outcomes can be achieved for both.

Lucy A. Stephenson, MBBS, BSC, MSC, MRCPsych, Department of Psychological Medicine, Institute of Psychiatry, Psychology and Neuroscience, King's College London, UK; Alastair J. D. Macdonald, MD, FRCPsych, Chair, Trust Outcomes Group, South UK; Alastair J. D. Macdonald, MD, FRCPsych, Chair, Trust Outcomes Group, South
London and Maudsley NHS Foundation Trust, and NIHR Maudsley Biomedical Research Centre, The Maudsley Hospital, London, UK; Gertrude Seneviratne, MBBS, MRCPsych Associate Clinical Director and Consultant Perinatal Psychiatrist, Perinatal Mental Health Services, South London \& Maudsley NHS Foundation, London, UK; Freddie Waites, BSC, MSc, Department of Psychosis Studies, Institute of Psychiatry, Psychology and Neuroscience, King's College London, UK; Susan Pawlby, MA, PhD, CPsychol, Department of Psychological Medicine, Institute of Psychiatry, Psychology and Neuroscience, King's College London, UK.

Correspondence: Susan Pawlby, Department of Psychological Medicine, Institute of Psychiatry, Psychology and Neuroscience, King's College London, De Crespigny Park, London SE5 8AF, UK. Email: susan.pawlby@kcl.acuk

First received 8 Dec 2017, final revision 8 Dec 2017, accepted 8 Jan 2018

\section{Acknowledgements}

We thank Afifa Ashfaque, Alessandra Biaggi, Rebecca Bind and Julia Tarrant for rating the video clips of mother-infant interaction using the CCl.

\section{References}

1 Bauer A, Pawlby S, Plant DT, King D, Pariante CM, Knapp M. Perinatal depression and child development: exploring the economic consequences from a South London cohort. Psychol Med 2015; 45(01): 51-61.

2 Bauer A, Parsonage M, Knapp M, lemmi V, Adelaja B. Costs of Perinatal Mental Health Problems. London School of Economics and Political Science, 2014.

3 National Institute for Health and Care Excellence. Antenatal and Postnatal Mental Health: Clinical Management and Service Guidance. NICE, 2014 (https://www.nice.org.uk/guidance/cg192/resources/antenatal-and-postnatalmental-health-clinical-management-and-service-guidance-pdf-35109869806789).

4 National Health Service England. Next Steps on the Five Year Forward View. NHSE, 2017 (https://www.england.nhs.uk/wp-content/uploads/2017/ 03/NEXT-STEPS-ON-THE-NHS-FIVE-YEAR-FORWARD-VIEW.pdf).

5 Murray L, Cooper PJ, Wilson A, Romaniuk H. Controlled trial of the short- and long-term effect of psychological treatment of post-partum depression:
2. Impact on the mother-child relationship and child outcome. Br J Psychiatry 2003; 182: 420-7.

6 Forman DR, O'Hara MW, Stuart S, Gorman LL, Larsen KE, Coy KC. Effective treatment for postpartum depression is not sufficient to improve the developing mother-child relationship. Dev Psychopathol 2007; 19(2): 585-602.

7 Stein A, Pearson RM, Goodman SH, Rapa E, Rahman A, McCallum M, et al. Effects of perinatal mental disorders on the fetus and child. Lancet 2014; 384 (9956): 1800-19.

8 Aldridge H, Born TB, Tinson A, Macinnes T. London's Poverty Profile 2015. Trust for London/New Policy Institute, 2015.

9 Kenny M, Conroy S, Pariante CM, Seneviratne G, Pawlby S. Mother-infant interaction in mother and baby unit patients: before and after treatment. J Psychiatr Res 2013; 47(9): 1192-8.

10 Overall JE, Gorham DR. The brief psychiatric rating scale. Psychol Rep 1962; 10: 799-812.

11 Shafer A. A meta-analysis of the brief psychiatric rating scale factor structure. Psychol Assess 2005; 17: 324-35.

12 Leucht ST, Kane JM, Kissling WE, Hamaan JO, Etschel EV, Engel RO. Clinical implications of brief psychiatric rating scale scores. Br J Psychiatry 2005; 187: 366.

13 Wing JK, Beevor AS, Curtis RH, Park SB, Hadden S, Burns A. Health of the Nation Outcome Scales (HONOS). research and development. Br J Psychiatry 1998; 172: $11-8$.

14 Stewart R, Soremekun M, Perera G, Broadbent M, Callard F, Denis M, et al. The South London and Maudsley NHS Foundation Trust Biomedical Research Centre (SLAM BRC) case register: development and descriptive data. BMC Psychiatry 2009; 9: 51.

15 Crittenden P. CARE-Index: Manual. Miami: Family Relations Institute, 2004.

16 Cohen J. Statistical Power Analysis for the Behavioural Sciences. Lawrence Earlbaum Associates, 1988.

17 McClelland R, Trimble P, Fox ML, Stevenson MR, Bell B. Validation of an outcome scale for use in adult psychiatric practice. Qual Health Care 2000; 9: 98-105.

18 Christl B, Reilly N, Yin C, Austin MP. Clinical profile and outcomes of women admitted to a psychiatric mother-baby unit. Arch Womens Ment Health 2015; 18(6): 805-16.

19 Kumar R, Marks M, Platz C, Yoshida K. Clinical survey of a psychiatric mother and baby unit: characteristics of 100 consecutive admissions. J Affect Disord 1995; 33(1): 11-22.

20 Salmon MP, Abel K, Webb R, Warburton AL, Appleby L. A national audit of joint mother and baby admissions to UK psychiatric hospitals: an overview of findings. Arch Womens Ment Health 2004; 7(1): 65-70.

21 Buist A, Minto B, Szego K, Samhuel M, Shawyer L, O'Connor L. Mother-baby psychiatric units in Australia - the Victorian experience. Arch Womens Ment Health 2004; 7(1): 81-7.

22 Glangeaud-Freudenthal NM. Mother-Baby psychiatric units (MBUs): nationa data collection in France and in Belgium (1999-2000). Arch Womens Ment Health 2004; 7(1): 59-64.

23 Salmon $\mathrm{M}$, Abel K, Cordingley L, Friedman T, Appleby L. Clinical and parenting skills outcomes following joint mother-baby psychiatric admission. Aust NZ $\mathrm{J}$ Psychiatry 2003; 37(5): 556-62.

24 Donabedian A. Evaluating the quality of medical care. Milbank Memorial Fund Q 1966; 44: 166-206.

25 Abel KM, Webb RT, Salmon MP, Wan MW, Appleby L. Prevalence and predictors of parenting outcomes in a cohort of mothers with schizophrenia admitted for joint mother and baby psychiatric care in England. J Clin Psychiatry 2005; 66(6): 781-9.

26 NHS Digital. Hospital Admitted Patient Care Activity 2016-2017. NHS Digital, 2017 (https://digital.nhs.uk/catalogue/PUB30098).

27 Schacht R, Meins E, Fernyhough C, Centifanti LC, Bureau JF, Pawlby S. Proof of concept of a mind-mindedness intervention for mothers hospitalized for severe mental illness. Dev Psychopathol 2017; 29(2): 555-64. 